\title{
Targeting ferroptosis in acute kidney injury
}

\author{
Lihua $\mathrm{Ni} \mathbb{D}^{1}$, Cheng Yuan $\mathbb{D}^{2 凶}$ and Xiaoyan Wu (D) ${ }^{1 凶}$
}

(c) The Author(s) 2022

Acute kidney injury (AKI) is a major public health problem with high incidence and mortality. As a form of programmed cell death (PCD), ferroptosis could be considered as a process of iron accumulation and enhanced lipid peroxidation. Recently, the fundamental roles of ferroptosis in AKI have attracted much attention. The network mechanism of ferroptosis in AKI and its roles in the AKI to chronic kidney disease (CKD) transition is complicated and multifactorial. Strategies targeting ferroptosis show great potential. Here, we review the research progress on ferroptosis and its participation in AKI. We hope that this work will provide clues for further studies of ferroptosis in AKI.

Cell Death and Disease (2022)13:182; https://doi.org/10.1038/s41419-022-04628-9

\section{FACTS}

- Ferroptosis is an iron- and reactive oxygen species (ROS)dependent lipid peroxidation that is different from the other forms of programmed cell death (PCD) at the morphological and biochemical levels.

- Ferroptosis participates in the occurrence and development of acute kidney injury (AKI).

- Numerous studies have demonstrated that strategies targeting ferroptosis can delay the progression of AKI.

\section{OPEN QUESTIONS}

- How can ferroptosis be detected?

- What is the interplay of mitochondria and lysosomes, the ER, and the Golgi in ferroptosis?

- How does Golgi stress induce ferroptosis?

- How does the complicated network mechanism regulate ferroptosis?

- What is the role of ferroptosis in the AKI to chronic kidney disease (CKD) progression?

- What is the interplay of ferroptosis and necroptosis in the progression of AKI?

\section{INTRODUCTION OF ACUTE KIDNEY INJURY (AKI)}

The prevalence of acute kidney injury (AKI) is increasing in hospitalized patients [1-3]. The incidence ranges from $10-15 \%$ in all hospitalizations and is as high as $50 \%$ in the intensive care unit (ICU) $[4,5]$. In addition, it demonstrated that AKI increase the potential risks of chronic kidney disease (CKD), cardiovascular disease, and end-stage renal disease (ESRD) [6-8]. Generally, several factors, such as ischemia, insufficiency of the circulating blood volume, nephrotoxic drugs, and urinary tract obstruction, lead to AKI. Apart from blood purification, there are few effective treatments for AKI. Untreated AKI would continue to cause further damage and is associated with a poor prognosis. Thus, exploring novel targets or drugs is desperately needed.

The pathogenesis of AKI is considered to involve nephrotoxicity, the inflammatory response, acute tubular hypoxia and necrosis, pericyte injury, and microvascular injury/dysfunction [9-13]. Recently, ferroptosis has been demonstrated to be involved in the pathogenesis and therapeutic strategies of AKI. Ferroptosis induced a first wave of death, triggering an inflammatory response that in turn contributed to the deterioration of renal function. And strategies targeting ferroptosis showed great therapeutic potential. Our study summarizes the current progress on ferroptosis and its roles in AKI.

\section{FERROPTOSIS AND CELL DEATH}

Ferroptosis (one center, two characteristics, and four aspects) The concept of ferroptosis was first proposed in 2012 by Dixon et al. [14]. The characteristics of ferroptosis have been studied for several years and can be summarized as one center, two characteristics, and four aspects [15-17]. One center refers to the accumulation of lipid reactive oxygen species (ROS). Two characteristics refer to the destruction of cellular antioxidants and enhanced levels of intracellular iron, which lead to the deposition of detrimental lipid ROS. Four aspects indicate metabolic disorder of iron, the amplified production of ROS and accumulation of lipid peroxide, and the consumption of glutathione peroxidase 4 (GPX4) and system $\mathrm{Xc}^{-}$(a cysteine/ glutamate antiporter system).

Morphologically, ferroptosis mainly manifests as shrinkage of mitochondria with the rupture of mitochondria membrane,

\footnotetext{
${ }^{1}$ Department of Nephrology, Zhongnan Hospital of Wuhan University, Wuhan 430071, China. ${ }^{2}$ Department of Gynecological Oncology, Zhongnan Hospital of Wuhan University, Wuhan 430071, China. ${ }^{\circledR}$ email: yuancheng_89@whu.edu.cn; wuxiaoyan_kid@163.com

Edited by Professor Boris Zhivotovsky
}

Received: 7 September 2021 Revised: 24 January 2022 Accepted: 9 February 2022

Published online: 24 February 2022 
enhanced membrane density, and reduction or disappearance of the mitochondrial crest [18]. Based on the morphological particularity of ferroptosis, transmission electron microscopy (TEM) is widely applied to observe ultrastructural changes [19].

Biochemically, ferroptosis mainly includes the consumption of glutathione (GSH) and the decreased activity of GPX4. In addition, the metabolism of iron, amino acids, and ROS is also associated with ferroptosis $[20,21]$.

\section{Comparisons of the common types of cell death}

Cell death can be classified into programmed cell death $(P C D$, such as ferroptosis, apoptosis, necroptosis, and autophagydependent cell death) and accidental cell death (such as necrosis) [22-25]. As an iron-dependent nonapoptotic cell death, ferroptosis is different from other cellular deaths (apoptosis, necrosis, and autophagy) [26-29]. The accumulation of free iron is a key initiator of ferroptosis. During ferroptosis, the cell membrane is destabilized, the cytoskeleton is rearranged, and proteostasis is disrupted. Green et al. suggested that ferroptosis could be best described as a cellular "sabotage", meaning that normal metabolic functions lead to cell death [29].

Apoptosis is the most common type of PCD. During apoptosis, cells shrink, the plasma membrane bubbles, and apoptotic bodies form. Abundant cellular proteases contribute to the disintegration of skeletons, membranes, and proteins [30-32]. We also consider apoptosis to be a process of self-killing.

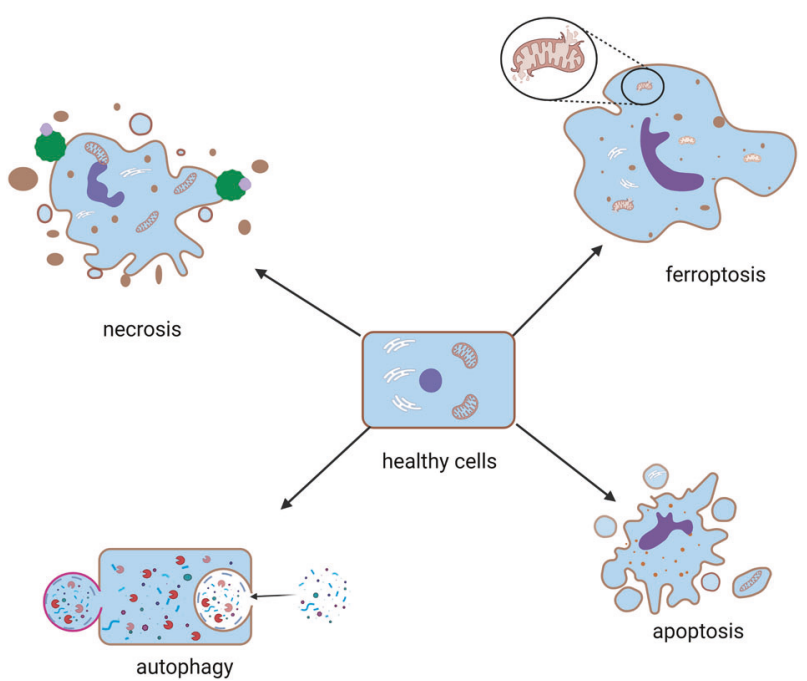

Fig. 1 Schematic representation of the main types of cell death. Cell death can be classified into programmed cell death (PCD, such as ferroptosis, apoptosis, and autophagy) and accidental cell death (such as necrosis).
Autophagy-dependent cell death is a natural degradation process of cellular contents and a rare kind of PCD [33-35]. During autophagy-dependent cell death, extensive vacuolization of the cytoplasm is formed; the integrity of the plasma membrane is lost; and sometimes, enlargement of the Golgi and ER is observed [36-38]. We also consider autophagy-dependent cell death to be a process of self-eating.

Necroptosis, a widely studied programmed necrosis, is mediated by the receptor-interacting protein kinase 1 (RIPK1), receptor-interacting protein kinase 3 (RIPK3), and mixed lineage kinase domain-like protein (MLKL). During necroptosis, the cytoplasm was swelling, the plasma membrane was raptured and the intracellular content was spilling.

Necrosis is a kind of unregulated cell death that comes from stress stimuli. During necrosis, the cell volume is increased, the integrity of the plasma membrane is damaged, and the cellular contents are lost [39-42]. Decay products recruit lymphocytes, leukocytes, and macrophages to phagocytose dead cells.

Above all, there are many differences among the various kinds of cell death (Fig. 1). Ferroptosis is different from the others in morphology and biochemistry. Iron-dependent lipid peroxidation is the most important feature indicating that ferroptosis is different from the other kinds of cell death.

\section{THE DETECTION OF FERROPTOSIS}

It is worth noting that to date, there is no specific method for the detection of ferroptosis. According to the features of ferroptosis, the most frequent types of detection can be divided into five parts: cell viability, cytotoxicity and death, levels of iron, ROS, and biomarkers and morphologic changes (Table 1). A cell counting kit-8 (CCK-8) assay can be applied to detect cell viability. Assay of lactate dehydrogenase (LDH) release was used to analyze cell cytotoxicity. Ferroptotic cellular death can be assessed by TUNEL assay. The detection of serum nonheme iron can be conducted using an iron/ TIBC reagent. The chromogen method can detect tissue nonheme iron. Malondialdehyde (MDA) and 4-hydroxynonenal (4HNE) can be used to evaluate iron-related lipid peroxidation. In addition, the levels of GSH and GPx4 can be measured to study the inhibition of antioxidants during ferroptosis. Detecting ferroptosis-associated biomarker proteins, such as nuclear respiratory factor 2 (NRF2), nuclear receptor coactivator 4 (NCOA4), heme oxygenase-1 (HO-1), GPX4, and heavy peptide ferritin (FTH), are conventional methods. As mentioned above, TEM can also be used to observe the ultrastructural changes during ferroptosis.

Generally, it is encouraged to perform five or more levels of methods together-in other words, the greater the better.

\section{REGULATORY MECHANISM OF FERROPTOSIS}

The mechanism of ferroptosis has been widely studied. System $\mathrm{Xc}^{-}, \mathrm{GPX} 4$, iron homeostasis, ROS, and lipid peroxidation are the most extensively studied mechanisms (Fig. 2).

Table 1. The measurement of ferroptosis.

\begin{tabular}{l} 
Detection \\
Cell viability, cytotoxicity, and death \\
Iron levels \\
\hline ROS levels and lipid peroxidation \\
Mitochondrial oxidative stress \\
Biomarker proteins \\
Morphology
\end{tabular}

\section{Methods}

CCK-8, LDH release, and TUNEL

Flow cytometry or confocal via Phen Green SK probes, chromogen method, Iron/TIBC reagent MDA, 4HNE, flow cytometry via BODIPY and DCFH-DA, JC-1, GSH

Mito TEMPO NRF2, NCOA4, HO-1, GPX4, FTH, COX2, PTGS2, ACSL4, NOX1, SLC7A11, metallothionein-1 TEM
References

$[136,137]$

[138]

[139-142]

Note: CCK-8 Cell Counting Kit, NRF2 nuclear respiratory factor 2, NCOA4 nuclear receptor coactivator 4, HO-1 heme oxygenase-1, FTH heavy peptide ferritin, TEM transmission electron microscopy, ACSL4 acyl-CoA synthetase long-chain family member 4, NOX1 NADPH oxidase activator 1, SLC7A11 cysteine/ glutamate antiporter solute carrier family 7 member 11. 


\section{System Xc}

As cysteine/glutamate reverse transporters expressed on the plasma membrane, system $\mathrm{Xc}^{-}$donates intracellular glutamate and takes extracellular cystine at a molar ratio of 1:1 [43]. System $\mathrm{Xc}^{-}$includes two subunits, light chain $\mathrm{xCT}$, and heavy chain 4F2hc. Inhibition of system $\mathrm{Xc}^{-}$contributes to decreased expression of GSH and different types of cell death. In addition, xCT is a major gene for the regulation of iron overload/ferroptosis via gene chip screening. Further studies have indicated that $\mathrm{xCT}$ attenuates ferroptosis during iron overload [44, 45]. Some agents, such as sorafenib, sulfasalazine, and glutamate, can trigger ferroptosis by blocking system $\mathrm{Xc}^{-}$[46].

Briefly, impairment of the system $\mathrm{Xc}^{-}$dependent antioxidant defense system contributes to oxidative damage and thus triggers ferroptosis. Erastin induces ferroptosis by blocking system $\mathrm{Xc}^{-}$ [46]. However, due to the lack of specificity and off-target effects, the development of therapies targeting $\mathrm{xCT}$ has been constrained [47]. Hence, finding a highly effective and specific xCT blocker is of vital importance.

\section{GPX4}

GPX4 is a key enzyme that facilitates GSH to counteract lipoxygenase (Alox) activity and phospholipid/cardiolipin oxidative events [48]. RSL3 binds and further inactivates GPX4 to induce ferroptosis by decreasing the antioxidant capacity and the accumulation of ROS [49]. GPX4 knockout in tubular cells leads to ferroptosis and massive cell death [50]. Yang et al. demonstrated that cells with downregulated expression of GPX4 were more sensitive to ferroptosis, while upregulated GPX4 inhibited ferroptosis [51]. In response to GPX4 inactivation and system $\mathrm{Xc}^{-}$ inhibition, cells undergoing ferroptosis participate in the irondependent decrease in polyunsaturated fatty acids (PUFAs) and the accumulation of L-ROS $[52,53]$. PUFAs contribute to the formation of lipid hydroperoxides (L-OOH). Iron and PUFAs give rise to enhanced toxic levels of L-ROS. Friedman Angeli et al. had proved the involvement of GPX4 activation in AKI [50]. And irisin could attenuate IRI-induced AKI through upregulating GPX4 [54]. The recent work showed that IRI-induced renal ferroptosis is regulated by an augmenter of liver regeneration (ALR), which is associated with the GSH-GPX4 system [55].

Simply put, RSL3 induces ferroptosis by directly inhibiting GPX4. Inactivated GPX4 leads to enhanced production of lipid-based

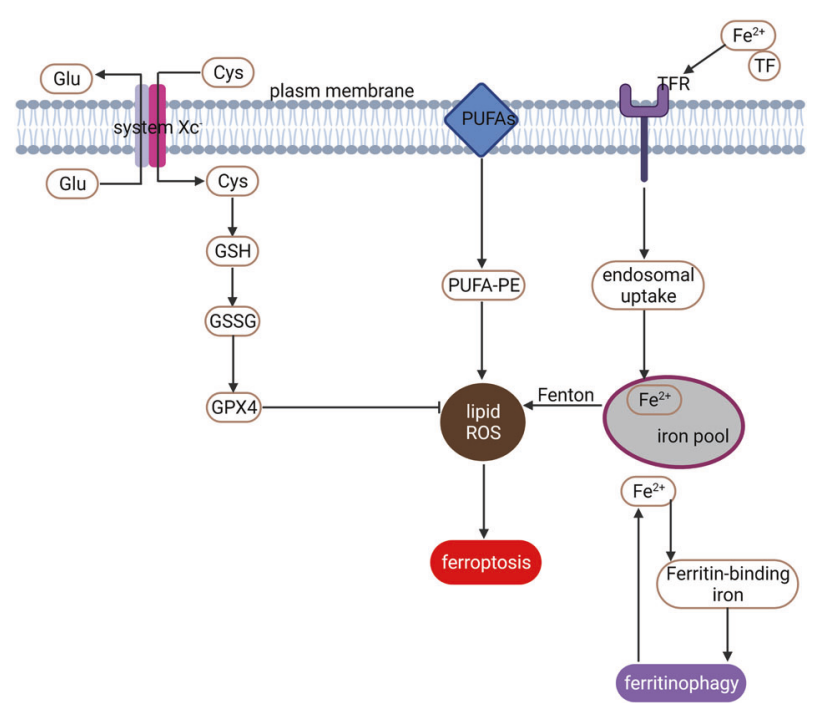

Fig. 2 Regulatory mechanism of ferroptosis. General mechanism of ferroptosis associated with System Xc-, GPX4, iron homeostasis, ROS, and lipid peroxidation.
ROS and lipid hydroperoxides, which ultimately induce ferroptosis [16].

\section{Iron homeostasis}

Ferroptosis is characterized by iron-dependent oxidative damage to some degree $[15,56]$. Excessive iron causes harm by generating ROS and regulating lipid peroxidation.

Physiologically, internal absorption- and erythrocyte degradationderived $\mathrm{Fe}^{2+}$ can be oxidized to $\mathrm{Fe}^{3+}$. $\mathrm{Fe}^{3+}$ can enter the cells by bonding to transferrin (TF), which forms the complex of "TF-Fe ${ }^{3+}-\mathrm{TFR} 1$ " by TF receptor 1 (TFR1). Then, the intracellular $\mathrm{Fe}^{3+}$ is further converted to $\mathrm{Fe}^{2+}$ and stored in the iron pool $[57,58]$.

Pathologically, excess $\mathrm{Fe}^{2+}$ can be oxidized to $\mathrm{Fe}^{3+}$ and exported by ferroportin (FPN), which increases the production of ROS through the Fenton reaction and ultimately induces ferroptosis. Ferritin is a main intracellular iron storage protein. Ferritin can be divided into ferritin light chain (FTL) and ferritin heavy chain (FTH). The FTL facilitates the iron nuclei formation, while the FTH promotes ferrous oxidase. Collectively, ferritin serves to iron storage and utilization. Inhibiting TFR1 can alleviate erastin-induced ferroptosis [59, 60]. Blocking iron response element-binding protein 2 (IREB2), the main transcription factor for iron hemostasis, can obviously alleviate erastin-induced expression of FTL and FTH. The role of ferritin in preventing sepsis-induced AKI had been aroused. More research about the functions of ferritins in sepsis-induced AKI might be encouraged [61].

Taken together, the disturbance of iron metabolism in favor of iron overload contributes to ferroptosis.

\section{ROS and lipid peroxidation}

Enhanced accumulation of ROS is one of the major causes of ferroptosis. Erastin treatment results in inhibiting system $\mathrm{Xc}^{-}$, which further initiates mitochondrial dysfunction, disturbances of cellular redox homeostasis, and the formation of lipid peroxides. All these combined actions motivate ferroptosis [17, 62].

Erastin increases the production of lipid ROS by inhibiting system $\mathrm{Xc}^{-}$, which ultimately induces ferroptosis [63]. RSL3 enhances lipid peroxidation by directly binding to GPX4, which ultimately triggers ferroptosis [53, 64]. Additionally, numerous lipophilic antioxidants, such as ferrostatin-1 (Fer-1), liproxstatin-1, $\beta$-carotene, and a-tocopherol, have been proven to suppress ferroptosis [65].

In summary, lipid metabolism is significantly associated with ferroptosis.

\section{Others}

As a proinflammatory form of PCD, ferroptosis can be regulated by several small molecules and genetic manipulation. Recent publications are summarized in Table 2.

The processes of AKI are very complicated. The extent to which ferroptosis participates in AKI still needs further research. The relationship between ferroptosis and iron hemostasis, ROS, and lipid peroxidation remained to be further addressed in AKI.

THE FUNDAMENTAL ROLES OF ORGANELLES IN FERROPTOSIS Organelles are important parts of cells that serve to maintain intracellular homeostasis. Recently, mitochondrial dysfunction, lysosome dysfunction, Golgi dysfunction, and ER dysfunction have been suggested to participate in the regulation of ferroptosis.

\section{Mitochondria}

Previously, mitochondria were well-known energy stations for cellular activities and were closely associated with the process of $P C D$. The roles of mitochondria in ferroptosis have attracted great attention [66-68]. First, ferroptosis causes irreversible morphological changes in mitochondria [69]. Ferroptosis is characterized by 
Table 2. Mediators or modulators of ferroptosis.

\begin{tabular}{|c|c|}
\hline Proteins & Name \\
\hline VDACs & voltage-dependent anion channels \\
\hline p53 & cellular tumor antigen $\mathrm{p} 53$ \\
\hline FSP1 & $\begin{array}{l}\text { ferroptosis suppressor protein } 1 \text { (a CoQ } \\
\text { oxidoreductase, which) }\end{array}$ \\
\hline Panx 1 & Pannexin 1 \\
\hline NRF2 & nuclear respiratory Factor 2 \\
\hline $\mathrm{CoQ}$ & coenzyme Q (a subtract of the oxidoreductase) \\
\hline HSPB1 & heat shock 27 kDa protein 1 \\
\hline VDR & Vitamin D receptor \\
\hline NOX & NADPH oxidase activator \\
\hline TfR1 & Transferrin receptor protein 1 \\
\hline ACSL4 & Acyl-CoA synthetase long-chain family member 4 \\
\hline CARS & Cysteinyl-tRNA synthetase \\
\hline MAPK & Mitogen-activated protein kinase 1 \\
\hline NCOA4 & Nuclear receptor coactivator 4 \\
\hline $15 \mathrm{LO}$ & 15-lipoxygenases \\
\hline SLC7A11 & $\begin{array}{l}\text { Cysteine/glutamate antiporter solute carrier family } \\
\text { member } 11\end{array}$ \\
\hline PEBP1 & Phosphatidylethanolamine-binding protein 1 \\
\hline miR-182-5p & microRNA-182-5p \\
\hline miR-378a-3p & microRNA-378a-3p \\
\hline
\end{tabular}

a reduced density of the mitochondrial membrane, a smaller volume of mitochondria, decreased mitochondrial cristae, and a ruptured mitochondrial outer membrane (OMM). Second, mitochondria play predominant roles in iron utilization and anabolic and catabolic pathways [56, 70]. Increased iron uptake and mobilization lead to the deposition of intracellular iron in ferroptosis. Third, mitochondria regulate energetic metabolism in ferroptosis [21, 71]. Temporary activation of aerobic respiration is associated with oxidative injury to mitochondria in ferroptosis. Mitochondrial ROS play significant roles in ferroptosis. Fourth, mitochondria-lipid metabolism play critical roles in ferroptosis [72]. Mitochondria play vital roles in ferroptosis, where their morphology and function are irreversibly injured. The electron microscopy observation exhibited more swollen mitochondria in renal tissues of cisplatin-induced AKI animals than the normal controls, which could be alleviated by Fer-1 administration [73]. Accordingly, the HK-2 cells subjected to I/R were characterized by the shrinking of the shape, disorganization, and a reduction of the mitochondrial crista [55]. Targeting the mitochondrial-associated metabolic pathway might be a potential therapeutic strategy for ferroptosis-related diseases.

\section{Lysosomes}

Lysosomes are well-known membrane-bound cell organelles that mediate the degradation of all kinds of biomolecules. Disordered lysosomes recruit undigested substances, which ultimately contribute to lysosomal storage dysfunction. Recently, researchers have pointed out that lysosomes play a significant role in the formation of ferroptosis [74-76]. First, lysosomes are the major storage location for iron [77]. The activity of lysosomes could affect intracellular iron provision by alleviating intracellular

\section{Mechanism}

Production of mitochondrial ROS and mitochondrial

References dysfunction

System $\mathrm{Xc}^{-}$inhibition

FSP1 can reduce phospholipid

$[147,148]$

[149]

Panx 1 negatively regulates lipid peroxidation through the MAPK pathway

NRF2 can bind to ARE elements in the promoter regions of the target genes

The CoQ oxidoreductase FSP1 acts parallel to GPX4 to inhibit ferroptosis

HSPB1 phosphorylation is negatively regulated and ironmediated in the production of lipid ROS

VDR mediates the transcription of GPX4

Produces ROS

Mediates iron metabolism

Regulates the synthesis of fatty acyl CoA

$[137,154]$

Involved in the trans-sulfuration and synthesis of GSH

Mediates cellular growth, survival, adhesion, and differentiation

Regulates iron metabolism

Catalyzes the formation of pro-ferroptotic $15-\mathrm{OOH}-\mathrm{AA}$ (HpETE)

$[163,164]$

Regulates the uptake of cysteine and the release of glutamate

Inhibits the Ras/MEK/ERK cascade

miR-182-5p correlates reversely with GPX4

miR-378a-3p correlates reversely with SLC7A11

transferrin transportation or autophagic degradation of ferritin. Second, lysosomes are the main resource of cellular ROS in chemical-induced ferroptosis [69, 78]. The destabilized membrane of lysosomes leads to the release of large amounts of intracellular ROS, which subsequently contributes to cell death, including ferroptosis. Third, lysosomes are the main subcellular structure for the autophagic degradation of protein aggregates $[79,80]$. Additionally, ferroptosis is often regarded as an autophagic cell death pathway. This was further confirmed by Gao, who found that autophagy inhibition greatly alleviated cysteine deprivation-induced ferroptosis [81]. Chen et al. suggested that legumain promotes ferroptosis in IRI-AKI. And the protective effects of legumain deficiency depend on its inhibition of lysosomal degradation [82]. However, direct evidence demonstrating ferroptosis as a consequence of autophagy is still lacking.

\section{Endoplasmic reticulum (ER)}

As a subcellular organelle, the ER participates in the synthesis and exportation of proteins and lipids [10, 83]. Excess accumulation of unfolded proteins leads to ER stress. Moderate ER stress maintains the balance of intracellular homeostasis. Persistent ER stress contributes to apoptosis and cell death [10].

Recent studies have demonstrated that ER stress is involved in ferroptosis [84-86]. Zhang et al. found enhanced renal ER stress and the activation of ferroptosis in animal models of IRI-AKI [54]. The roles of ER stress in ferroptosis could be summarized as follows: first, ferroptosis inducers (erastin) trigger ferroptosis and ER stress together [87, 88]. Activated ER stress in turn inhibits ferroptosis and promotes drug resistance in a variety of tumors. Hence, the combined use of ferroptosis inducers and ER stress 

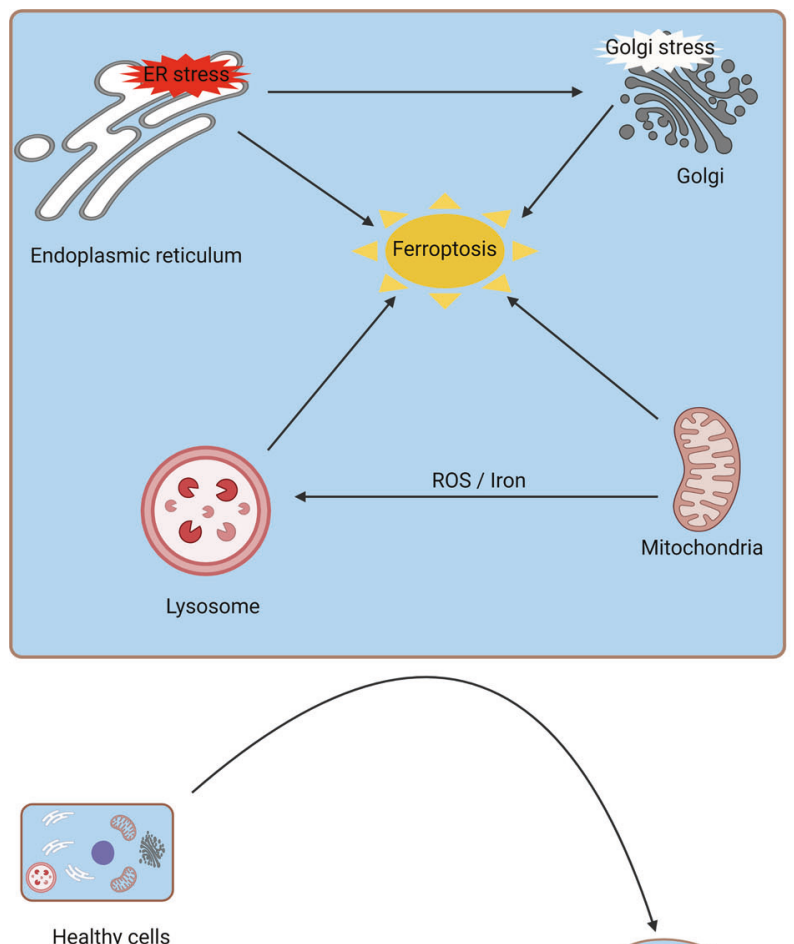

Healthy cells

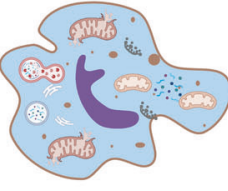

Ferroptosis

Fig. 3 The interplay between mitochondria, lysosomes, the ER, and the Golgi in ferroptosis. Mitochondria and lysosomes together participate in iron metabolism, which induces ferroptosis. In addition, mitochondrial ROS can be taken up by lysosomes, ultimately leading to ferroptosis. ER stress can induce the function of the Golgi, which regulates ferroptosis.

inhibitors in the treatment of cancer might be of great significance. Second, ER stress accelerates ferroptosis and worsens disease progression in some pathological conditions [89, 90]. Hence, the internal regulatory mechanism between ER stress and ferroptosis still needs further research. Third, ER stress is involved in the molecular crosstalk between ferroptosis and apoptosis [91]. The combined treatment of erastin or artesunate (ART) and tumor necrosis factor-related apoptosis-inducing ligand (TRAIL) can trigger apoptosis but not ferroptosis [91]. Erastin treatment alone does not lead to apoptosis. This result suggested that the relationship between ferroptosis and ER stress might be anergic or uncertain. ER stress might be the bridge between ferroptosis and apoptosis.

\section{Golgi stress}

The participation of Golgi stress in ferroptosis has rarely been studied [92]. First, Golgi-dispersing agents, including BFA, AG1478/tyrphostin, AMF-26, and GCA, induce ferroptosis $[92,93]$. Second, Golgi stress leads to a decreased intracellular glutathione pool, accumulation of lipid peroxides, and regulation of ferroptosis-associated signaling [92]. Third, iron chelators, antioxidants, overexpression of GPX4, and ACSL4 inhibition can prevent Golgi stress-induced ferroptosis [92]. Fourth, inhibitors of ferroptosis play beneficial effects on the function and morphology of the Golgi after Golgi stress inhibition administration [92].
Previously, studies on Golgi stress and ferroptosis mainly focused on cancer progression [94]. However, evidence in AKI is missing and still needs further research.

\section{The interplay between mitochondria, lysosomes, ER, and Golgi in ferroptosis}

Studies on organelle regulation have attracted great attention, especially in the field of ferroptosis $[95,96]$. As we mentioned above, there is a close interplay between mitochondria, lysosomes, the ER, and the Golgi in ferroptosis (Fig. 3). First, mitochondria and lysosomes together participate in iron metabolism, which is the main mechanism of ferroptosis induction $[77,97]$. Second, mitochondrial ROS can be taken up by lysosomes [69]. Excessive intracellular ROS lead to ferroptosis. Third, the capacity of the Golgi apparatus is modified according to ER stress [98]. However, the mechanisms of the organelle stress response are largely unclear. Investigations into the basic mechanisms of organelle regulation should receive much attention.

\section{FERROPTOSIS-RELATED BIOLOGICAL RESPONSE IN AKI Necroptosis}

Necroptosis is a common feature in AKI. Cells undergoing necroptosis exhibit disrupted cell membranes, which is the major difference from necrosis. The RIPK3 and its substrate MLKL play critical roles in the formation of necroptosis [99-101]. In acute ischemic kidney injury, the expression of acyl-CoA synthetase long-chain family member 4 (ACSL4, a key enzyme in ferroptosis) is increased and is correlated with the severity of renal damage $[82,102]$. Using CRISPR/Cas9 technology, Muller et al. found that knockout of Acs/4 conferred protection from erastin- and RSL3induced cell death in vitro [103]. Additionally, deletion of $M L K L$ restrained susceptibility to necroptosis. Ferroptosis and necroptosis are alternatives in some cases, which means that resistance to one pathway sensitizes cells to death via the other pathway [103]. Diego et al. demonstrated that ferroptosis, but not necroptosis, is the major cause of cell death in folic acid-induced AKI [104]. Enhanced ferroptosis promotes the renal inflammatory response, which aggravates renal insufficiency. While, in other cases, ferroptosis and necroptosis act in a synergic or sequential fashion.

Collectively, one regulated necrosis pathway compensates for another when either ferroptosis or necroptosis is comprised.

\section{Autophagy}

Autophagy is a dynamic intracellular degradation process that includes the formation and maturation of some membrane structures, such as autophagosomes, phagophores, and autolysosomes. Classical ferroptosis activators (such as erastin and RSL3) could increase autophagic flux in AKI $[82,105,106]$. Appropriate autophagy facilitates cell survival, while excessive autophagy might induce ferroptosis.

Ferritinophagy can regulate cellular iron through the autophagic degradation of ferritin [107]. Nuclear receptor coactivator 4 (NCOA4)-mediated ferritinophagy promotes iron accumulation in ferroptosis. In addition, RAB7A (a member of the RAS oncogene family)-mediated lipophagy, SQSTM1-mediated clockophagy and heat shock protein 90 (HSP90)-mediated chaperone-mediated autophagy (CMA) can induce ferroptosis by promoting lipid peroxidation.

Numerous studies have demonstrated that autophagy aggravates ferroptosis [108-110]. The mechanisms could be summarized as followed: First, autophagy generates lysosomal ROS. Second, autophagy leads to GSH depletion. Third, autophagy enhances lysosome cell death. Fourth, autophagy triggers lipid peroxidation. Fifth, autophagy recruits labile iron. While the crosstalk between autophagy and ferroptosis was complicated [111]. Recently, studies have also pointed out that both ferroptosis inducers and autophagy inducers facilitated the formation of 


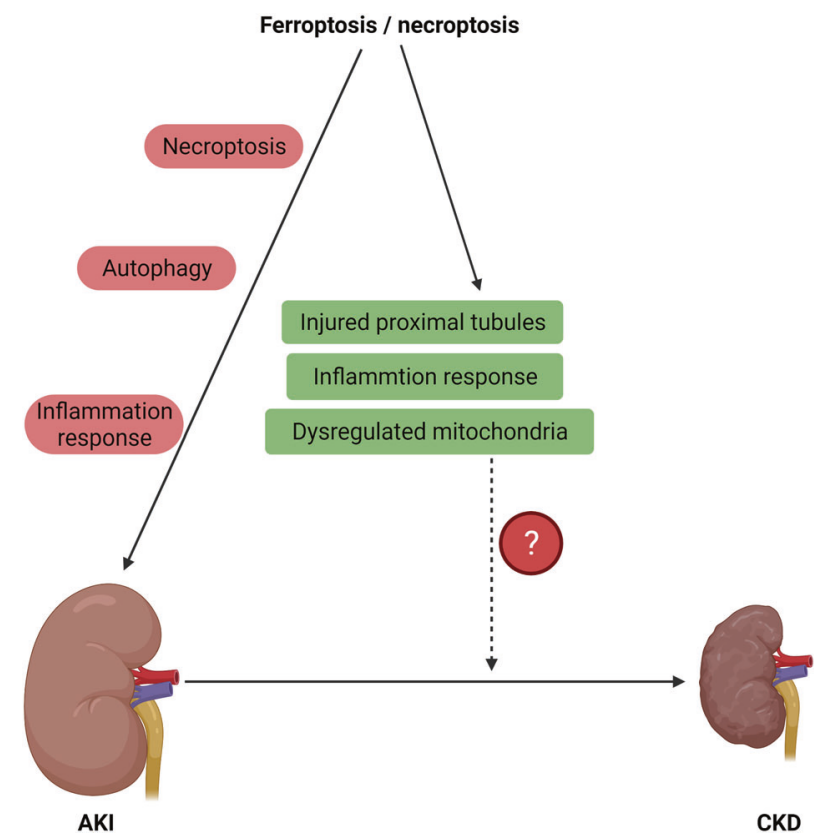

Fig. 4 The roles of ferroptosis in AKI and the AKI to CKD transition. Ferroptosis induces necroptosis, autophagy, and inflammatory responses, which lead to the progression of AKI. In some cases, ferroptosis and necroptosis act in a synergic or sequential fashion. In addition, targeting ferroptosis can alleviate tubular injury, mitochondrial function, and the inflammatory response, which further contribute to the AKI to CKD transition. However, direct evidence of ferroptosis in the AKI to CKD transition is lacking.

autophagosomes via activation ER stress. Enhanced ER stress contributed to the activation of autophagy [109]. That's to say, ferroptosis might also induce autophagy by ER stress. In addition, it had demonstrated selective autophagy or regulators of autophagic machinery in enhancing ferroptosis [107].

Collectively, autophagy is a lysosome-dependent cellular degradation process. Ferroptosis is an iron-dependent oxidative cell death pathway. In some conditions, ferroptosis has long been considered a form of autophagy-dependent cell death. Consequently, controlling the activity of autophagy during ferroptosis is of vital importance. However, the relationship between autophagy and ferroptosis is still controversial, which needs further research.

\section{Inflammation response}

Emerging evidence has suggested the positive roles of ferroptosis in inflammation through immunogenicity $[112,113]$. Ferroptosis in AKI can lead to the release of damage-associated molecular patterns (DAMPs) and alarmins, which further alert the innate immune system and shape the inflammatory response to cell death. Inhibitors of ferroptosis have anti-inflammatory effects in the process of AKI. The infiltration of neutrophils and the release of proinflammatory cytokines were alleviated by ferrostatin-1 (Fer-1, a ferroptosis inhibitor) in a mouse model of oxalate-induced AKI [114].

It should be noted that ferroptosis might be a double-edged sword. Therefore, it is of vital importance to determine the effects of ferroptosis on proinflammatory and anti-inflammatory responses.

\section{Interplay between ferroptosis, necroptosis, and inflammation in AKI}

Ferroptosis is an early event that induces an inflammatory response in AKI [115], and necroptosis is a secondary wave that maintains and amplifies renal disorders. Ferroptosis occurs before the diagnosis of AKI. Hence, targeting ferroptosis could be beneficial for early prevention in AKI, while targeting necroptosis could be useful for further therapeutic intervention in AKI. While the process of renal IRI is very complex. It was proved that necroptosis and ferroptosis were synchronized renal tubular cell death in renal IRI [114]. Hence, the relationship between necroptosis and ferroptosis still needs further research.

\section{FERROPTOSIS IN THE AKI TO CHRONIC KIDNEY DISEASE (CKD) PROGRESSION}

A significant number of AKI survivors can progress to chronic kidney disease (CKD) and further to end-stage renal disease (ESRD). It was demonstrated that $\sim 50 \%$ of hospital-associated AKI patients who survived AKI were newly diagnosed with CKD during a median follow-up period of 3.3 years [116]. Injured proximal tubules, dysregulated mitochondria, recruitment of immune cells, inflammatory responses, and heterogeneous backgrounds were described as the mechanisms of maladaptive repair after renal tubule damage [117]. Previous studies have suggested that targeting ferroptosis could prevent tubular injury, regulate the integrity of mitochondria, and alleviate inflammation via immune cells in rodent models of diabetic nephrology or AKI (Fig. 4) [118-121]. However, direct evidence about ferroptosis in the progression of AKI to CKD is lacking, which might be a hot point of research in the future.

\section{FERROPTOSIS IN SEVERAL RODENT MODELS OF AKI}

Emerging evidence has demonstrated the relationship between ferroptosis and AKI in vivo and in vitro. However, the specific regulatory mechanisms of ferroptosis in AKI are still largely unclear. Animal models for the investigation of ferroptosis are indispensable for further research [122].

\section{IRI-induced AKI}

$\mathrm{IRI}$, the most common reason for AKI, is characterized by a sudden pause in the blood supply to renal tissue and reoxygenation after blood flow is restored. AKI usually occurs due to clinical conditions, such as bleeding, dehydration, postoperative hypoperfusion, sepsis, and shock. Initially, apoptosis was thought to be the major kind of cell death in ischemic injury. In an in-depth study, necroptosis was regarded as the main cause of ischemic damage in the kidney and heart $[123,124]$. Recent observations indicated that ferroptosis could be dominant in ischemic injury. In a clinical study, low levels of early intraoperative iron-binding proteins exhibited a damaged ability to rapidly process catalytic iron released during extracorporeal circulation, which further contributed to renal injury in AKI [125]. In IRI-induced mice, ferrostatin administration alleviated the function and organ damage [114]. In isolated tubule cells, ferroptosis inhibitors protected against hypoxic injury [126].

\section{Nephrotoxicity-induced AKI}

Administration of nephrotoxic drugs is a common cause of renal injury and is widely applied for AKI research.

Folic acid (FA) triggers $A K I$ in animals. A certain dose of FA might induce crystals in the renal lumen, while high doses of FA might further damage the tubular epithelium [127]. High levels of renal lipid peroxidation were observed in a mouse model of FAAKI [104]. Pretreatment with Fer-1 alleviated the function and structure of the kidneys [104]. Interestingly, blocking necrotic apoptosis or apoptosis at the pharmacological or genetic level could not similarly reverse renal damage [104]. Thus, ferroptosis might be the major mechanism for regulatory necrosis in FA-AKI.

Cisplatin is a well-acknowledged antitumor agent. The feature of nephrotoxicity has attracted great concern [73, 128, 129]. Through experiments with a cisplatin-induced AKI model in vitro and in vivo, Baliga et al. suggested that cisplatin treatment led to increased expression of non transferrin-bound iron [130]. Additionally, deferoxamine (a chelating agent for removing excess 
iron) treatment significantly improved the functional and histological deterioration in cisplatin-induced AKI [131, 132].

Severe acute pancreatitis (SAP)-induced AKI is common in the clinic. AKI leads to drastic increases in mortality associated with SAP $[11,133]$. The mechanism by which SAP contributes to AKI is still under investigation. It has been demonstrated that the production of inflammatory mediators and various cytokines and

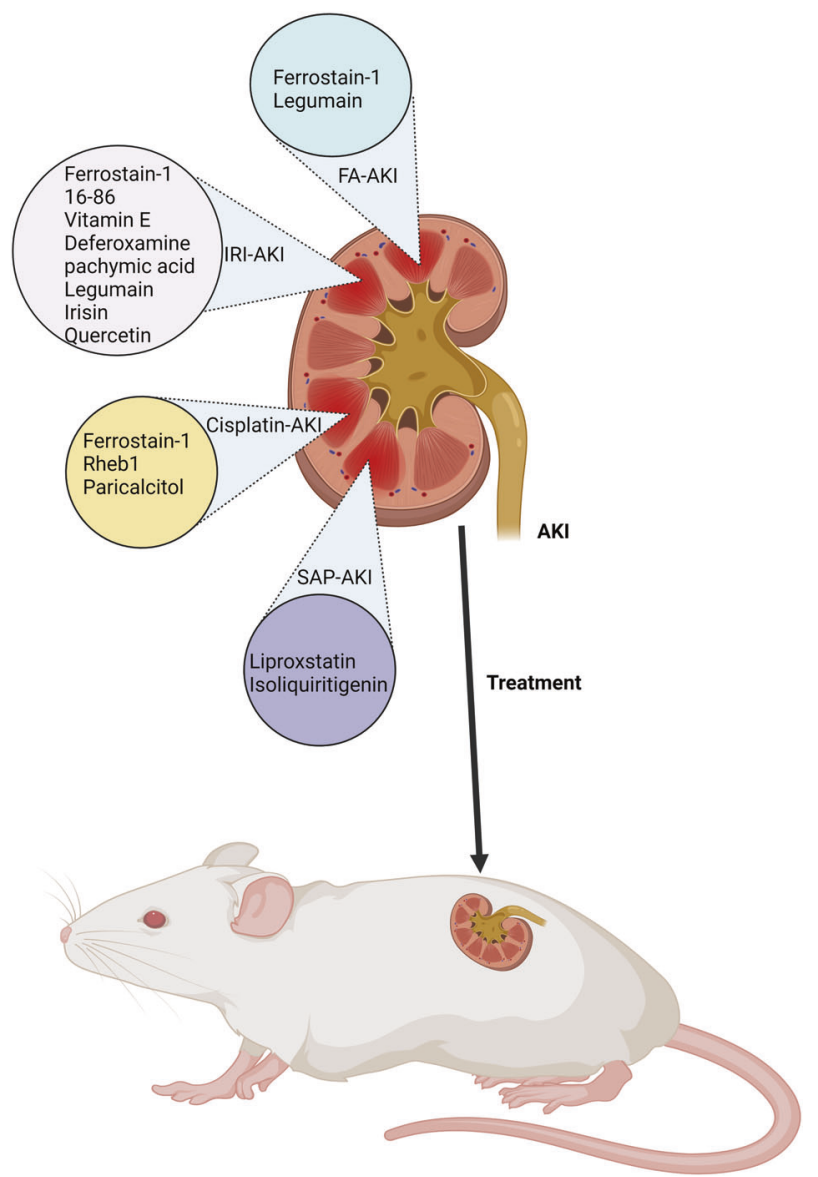

Fig. 5 Ferroptosis-targeted treatment in AKI. In rodent models of AKI, pharmacological inhibitors of ferroptosis can be employed to alleviate ferroptosis in AKI. the accumulation of ROS are involved in the process of SAP-AKI. The SAP-AKI model was established by perfusion of sodium taurocholate (5\%) into the biliopancreatic duct in rats. Ma et al. suggested the participation of ferroptosis in a rat model of SAPAKI, and ferroptosis was associated with renal damage [134].

Here, we briefly summarized the commonly used rodent models for the study of AKI (Fig. 5). Each model has its advantages and disadvantages. The IRI models seem to be the most popular and economic choices. Except for IRI, nephrotoxic models are suitable for the study of basic and common mechanisms of ferroptosis in AKI.

\section{PHARMACOLOGICAL ADVANCES REGARDING FERROPTOSIS IN AKI}

From a therapeutic point of view, pharmacological inhibitors of ferroptosis can be employed to alleviate ferroptosis in AKI [135]. These include inhibitors of system $\mathrm{Xc}^{-}, \mathrm{GPX} 4$, iron chelators, lipophilic antioxidants, and liproxstatins, which inhibit lipid peroxidation and are summarized in Table 3. How much benefits could we obtain from ferroptosis-targeting strategies in AKI, and even in AKI to CKD still need more in-depth studies. More comprehensive research on ferroptosis in the field of AKI to CKD is encouraged to broaden our understanding and techniques against renal damage.

\section{CONCLUSION}

Ferroptosis is a newly discovered PCD that is involved in lipid peroxidation and iron accumulation. It is different from other forms of cell death, such as apoptosis, necrosis, and autophagy, in terms of its distinguishable morphology, biochemistry, and genetics. The current studies summarized the introduction, detection, and mechanism of ferroptosis. The levels of iron and lipid oxidases are the most important features for the measurement of ferroptosis. The mechanism of ferroptosis mainly involves four processes: GPX4, system $\mathrm{Xc}^{-}$, iron metabolism, ROS, and lipid peroxidation. In addition, ferroptosis can be regulated by several small molecules and genetic manipulation, weaving a puzzling and intricate relationship network.

The participation of ferroptosis in AKI has been identified. The roles and mechanism of ferroptosis in the progression of AKI and therapeutic strategies targeting ferroptosis in AKI have been discussed in our review. To better understand ferroptosis in AKI, some animal models have been established, such as IRI-AKI and

Table 3. Ferroptosis-targeted therapies in AKI.

\begin{tabular}{|c|c|c|c|}
\hline Agents & Target & Diseases and models & References \\
\hline Ferrostatin-1 & Inhibits lipid peroxidation & IRI-AKI, cisplatin-AKI, FA-AKI & {$[104,168,170]$} \\
\hline $16-86$ & Inhibits lipid peroxidation & IRI-AKI & [114] \\
\hline Vitamin E & Lipophilic antioxidant & IRI-AKI & [171] \\
\hline Deferoxamine & Iron chelator & IRI-AKI & [172] \\
\hline Rosiglitazone & Inhibits ACSL4 & $\mathrm{GPX}^{-1-}$ mice & [173] \\
\hline Liproxstatin & Inhibits lipid peroxidation & $\mathrm{GPX}^{-1-}$ mice, SAP-induced AKI & {$[50,134]$} \\
\hline Legumain & Facilitates chaperone-mediated autophagy of GPX4 & IRI-AKI, FA-AKI & [82] \\
\hline Paricalcitol & Upregulates GPX4 & Cisplatin-AKI & [73] \\
\hline Isoliquiritigenin & upregulates the system $\mathrm{Xc}^{-}$and GPX4 & SAP-AKI & [176] \\
\hline Irisin & Upregulates GPX4 & IRI-AKI & [54] \\
\hline
\end{tabular}

Note: IRI-AKI ischemia and reperfusion injury-induced acute kidney injury, FA-AKI folic acid-induced acute kidney injury. Rheb1 Ras homolog enriched in brain. 
nephrotoxicity-AKI. From a therapeutic point of view, pharmacological treatment targeting ferroptosis could be conducted to alleviate ferroptosis in AKI.

\section{PERSPECTIVES}

The discovery of ferroptosis in AKI is still new, and associated research is greatly needed. Several issues should be addressed in future studies.

First, the current studies on the roles of ferroptosis in AKI came from in vivo and in vitro studies. More reliable animal models needed to be explored. Clinical studies are also encouraged.

Second, methods for the easy and reliable detection of ferroptosis are still lacking. Currently, the determination method is intrinsic, nonspecific, and time-consuming. Therefore, it is necessary to explore highly accurate and specific methods.

Third, current studies on ferroptosis have mainly focused on cancers. Mechanistic investigation of ferroptosis in AKI would have a good academic market, especially in the progression of AKI to CKD. In addition, ferroptosis participates in several renal diseases, such as diabetic nephrology and CKD.

Fourth, as a distinguishable PCD, ferroptosis occurs before the diagnosis of AKI. Early and precise detection of ferroptosis might be a novel biomarker for the diagnosis of $A K I$ and might be useful in clinical applications.

Although the above obstacles do exist and should be addressed in future studies, we believe that focusing on ferroptosis is essential and promising. Some attention, as well as increasing hope, should be anticipated by clinicians as researchers perform large-scale studies regarding ferroptosis.

\section{DATA AVAILABILITY}

The data used to support the findings of this study are available from the corresponding author upon request.

\section{REFERENCES}

1. Mehta RL, Pascual MT, Soroko S, Savage BR, Himmelfarb J, Ikizler TA, et al. Spectrum of acute renal failure in the intensive care unit: the PICARD experience. Kidney Int. 2004;66:1613-21.

2. Hsu RK, McCulloch CE, Dudley RA, Lo LJ, Hsu CY. Temporal changes in incidence of dialysis-requiring AKI. JASN. 2013;24:37-42.

3. Waikar SS, Curhan GC, Wald R, McCarthy EP, Chertow GM. Declining mortality in patients with acute renal failure, 1988 to 2002. JASN. 2006;17:1143-50.

4. Al-Jaghbeer M, Dealmeida D, Bilderback A, Ambrosino R, Kellum JA. Clinical decision support for in-hospital AKI. JASN. 2018;29:654-60.

5. Hoste EA, Bagshaw SM, Bellomo R, Cely CM, Colman R, Cruz DN, et al. Epidemiology of acute kidney injury in critically ill patients: the multinational AKI-EPI study. Intensive Care Med. 2015;41:1411-23.

6. Coca SG, Singanamala S, Parikh CR. Chronic kidney disease after acute kidney injury: a systematic review and meta-analysis. Kidney Int. 2012;81:442-8.

7. Chawla LS, Kimmel PL. Acute kidney injury and chronic kidney disease: an integrated clinical syndrome. Kidney Int. 2012;82:516-24.

8. Venkatachalam MA, Weinberg JM, Kriz W, Bidani AK. Failed tubule recovery, AKICKD transition, and kidney disease progression. JASN. 2015;26:1765-76.

9. Ferenbach DA, Bonventre JV. Acute kidney injury and chronic kidney disease: from the laboratory to the clinic. Nephrol Ther. 2016;12:S41-48.

10. Ni L, Yuan C, Wu X. Endoplasmic reticulum stress in diabetic nephrology: regulation, pathological role, and therapeutic potential. Oxid Med Cell Longev. 2021;2021:7277966.

11. Neyra JA, Chawla LS. Acute kidney disease to chronic kidney disease. Crit Care Clin. 2021;37:453-74.

12. Pesce F, Stea ED, Rossini M, Fiorentino M, Piancone F, Infante B, et al. Glomerulonephritis in AKI: from pathogenesis to therapeutic intervention. Front Med (Lausanne). 2020;7:582272.

13. Pickkers $P$, Darmon $M$, Hoste $E$, Joannidis $M$, Legrand $M$, Ostermann $M$, et al. Acute kidney injury in the critically ill: an updated review on pathophysiology and management. Intensive Care Med. 2021;47:835-50.
14. Dixon SJ, Lemberg KM, Lamprecht MR, Skouta R, Zaitsev EM, Gleason CE, et al. Ferroptosis: an iron-dependent form of nonapoptotic cell death. Cell. 2012;149:1060-72.

15. Li J, Cao F, Yin HL, Huang ZJ, Lin ZT, Mao N, et al. Ferroptosis: past, present and future. Cell Death Dis. 2020;11:88.

16. Yang WS, Stockwell BR. Ferroptosis: death by lipid peroxidation. Trends Cell Biol. 2016;26:165-76.

17. Latunde-Dada GO. Ferroptosis: role of lipid peroxidation, iron and ferritinophagy. Biochim Biophys Acta Gen Subj. 2017;1861:1893-1900.

18. Otasevic V, Vucetic M, Grigorov I, Martinovic V, Stancic A. Ferroptosis in different pathological contexts seen through the eyes of mitochondria. Oxid Med Cell Longev. 2021;2021:5537330.

19. Miyake S, Murai S, Kakuta S, Uchiyama Y, Nakano H. Identification of the hallmarks of necroptosis and ferroptosis by transmission electron microscopy. Biochem Biophys Res Commu. 2020;527:839-44.

20. Chen X, Comish PB, Tang D, Kang R. Characteristics and biomarkers of ferroptosis. Front Cell Dev Biol. 2021;9:637162.

21. Zheng J, Conrad M. The metabolic underpinnings of ferroptosis. Cell Metab. 2020;32:920-37.

22. Obeng E. Apoptosis (programmed cell death) and its signals-a review. Braz J Biol. 2021;81:1133-43.

23. Bedoui S, Herold MJ, Strasser A. Emerging connectivity of programmed cell death pathways and its physiological implications. Nat Rev Mol Cell Biol. 2020;21:678-95.

24. Wu J, Ye J, Kong W, Zhang S, Zheng Y. Programmed cell death pathways in hearing loss: a review of apoptosis, autophagy and programmed necrosis. Cell Prolif. 2020;53:e12915.

25. Zhivotovsky B. Programmed cell death: historical notes from Russia. Biochem (Mosc). 2020;85:1127-33.

26. Djulbegovic MB, Uversky VN. Ferroptosis-an iron-and disorder-dependent programmed cell death. Int J Biol Macromol. 2019;135:1052-69.

27. Zhou SY, Cui GZ, Yan XL, Wang X, Qu Y, Guo ZN, et al. Mechanism of ferroptosis and its relationships with other types of programmed cell death: insights for potential interventions after intracerebral hemorrhage. Front Neurosci. 2020;14:589042.

28. Kajarabille N, Latunde-Dada GO. Programmed cell-death by ferroptosis: antioxidants as mitigators. Int J Mol Sci. 2019;20:4968.

29. Green DR, Victor B. The pantheon of the fallen: why are there so many forms of cell death? Trends Cell Biol. 2012;22:555-6.

30. Elmore S. Apoptosis: a review of programmed cell death. Toxicol Pathol. 2007;35:495-516.

31. Sukumaran P, Nascimento Da Conceicao V, Sun Y, Ahamad N, Saraiva LR, Selvaraj $S$, et al. Calcium signaling regulates autophagy and apoptosis. Cells. 2021;10:2125.

32. Huang R, Hui Z, Wei S, Li D, Li W, Daping W, et al. IRE1 signaling regulates chondrocyte apoptosis and death fate in the osteoarthritis. J Cell Physiol. 2021. https://doi.org/10.1002/jcp.30537.

33. Chen XC, Li ZH, Yang C, Tang JX, Lan HY, Liu HF. Lysosome depletion-triggered autophagy impairment in progressive kidney injury. Kidney Dis (Basel). 2021;7:254-67.

34. Faruk MO, Ichimura Y, Komatsu M. Selective autophagy. Cancer Sci. 2021;112:3972-8.

35. Galluzzi L, Vitale I, Aaronson SA, Abrams JM, Adam D, Agostinis P, et al. Molecular mechanisms of cell death: recommendations of the Nomenclature Committee on Cell Death 2018. Cell Death Differ. 2018;25:486-541.

36. Guo R, Wang H, Cui N. Autophagy regulation on pyroptosis: mechanism and medical implication in sepsis. Mediators Inflamm. 2021;2021:9925059.

37. Tan S, Chen S. The mechanism and effect of autophagy, apoptosis, and pyroptosis on the progression of silicosis. Int J Mol Sci. 2021;22:8110.

38. Patra S, Praharaj PP, Klionsky DJ, Bhutia SK. Vorinostat in autophagic cell death: a critical insight into autophagy-mediated, -associated and -dependent cell death for cancer prevention. Drug Disco Today. 2022;27:269-79.

39. Lu Y, Zhou F, Luo Q. [Research progress on regulated necrosis in acute kidney injury]. Zhonghua wei zhong bing ji jiu yi xue. 2021;33:885-8.

40. D'Arcy MS. Cell death: a review of the major forms of apoptosis, necrosis and autophagy. Cell Biol Int. 2019;43:582-92.

41. Saleem S. Apoptosis, autophagy, necrosis and their multi galore crosstalk in neurodegeneration. Neuroscience. 2021;469:162-74.

42. Nikoletopoulou V, Markaki M, Palikaras K, Tavernarakis N. Crosstalk between apoptosis, necrosis and autophagy. Biochim Biophys Acta. 2013;1833:3448-59.

43. Liu MR, Zhu WT, Pei DS. System Xc(-): a key regulatory target of ferroptosis in cancer. Invest N. Drugs. 2021;39:1123-31.

44. Wang H, An P, Xie E, Wu Q, Fang X, Gao H, et al. Characterization of ferroptosis in murine models of hemochromatosis. Hepatology. 2017;66:449-65. 
45. Ashraf A, Jeandriens J, Parkes HG, So PW. Iron dyshomeostasis, lipid peroxidation and perturbed expression of cystine/glutamate antiporter in Alzheimer's disease: evidence of ferroptosis. Redox Biol. 2020;32:101494.

46. Zhao $\mathrm{Y}$, Li Y, Zhang R, Wang F, Wang $\mathrm{T}$, Jiao $\mathrm{Y}$. The role of erastin in ferroptosis and its prospects in cancer therapy. Onco Targets Ther. 2020;13:5429-41.

47. Liu L, Liu R, Liu Y, Li G, Chen Q, Liu X, et al. Cystine-glutamate antiporter xCT as a therapeutic target for cancer. Cell Biochem Funct. 2021;39:174-9.

48. Conrad M, Friedmann Angeli JP. Glutathione peroxidase 4 (Gpx4) and ferroptosis: what's so special about it? Mol Cell Oncol. 2015;2:e995047.

49. Yang WS, SriRamaratnam R, Welsch ME, Shimada K, Skouta R, Viswanathan VS, et al. Regulation of ferroptotic cancer cell death by GPX4. Cell. 2014;156:317-31.

50. Friedmann Angeli JP, Schneider M, Proneth B, Tyurina YY, Tyurin VA, Hammond $\mathrm{VJ}$, et al. Inactivation of the ferroptosis regulator $\mathrm{Gpx} 4$ triggers acute renal failure in mice. Nat Cell Biol. 2014;16:1180-91.

51. Yang WS, Stockwell BR. Synthetic lethal screening identifies compounds activating iron-dependent, nonapoptotic cell death in oncogenic-RAS-harboring cancer cells. Chem Biol. 2008;15:234-45.

52. Yu Y, Yan Y, Niu F, Wang Y, Chen X, Su G, et al. Ferroptosis: a cell death connecting oxidative stress, inflammation and cardiovascular diseases. Cell Death Disco. 2021;7:193.

53. Shui S, Zhao Z, Wang H, Conrad M, Liu G. Non-enzymatic lipid peroxidation initiated by photodynamic therapy drives a distinct ferroptosis-like cell death pathway. Redox Biol. 2021;45:102056.

54. Zhang J, Bi J, Ren Y, Du Z, Li T, Wang T, et al. Involvement of GPX4 in irisin's protection against ischemia reperfusion-induced acute kidney injury. J Cell Physiol. 2021;236:931-45.

55. Huang LL, Liao XH, Sun H, Jiang X, Liu Q, Zhang L. Augmenter of liver regeneration protects the kidney from ischaemia-reperfusion injury in ferroptosis. $J$ Cell Mol Med. 2019;23:4153-64.

56. Battaglia AM, Chirillo R, Aversa I, Sacco A, Costanzo F. Biamonte F. Ferroptosis and cancer: mitochondria meet the "iron maiden" cell death. Cells. 2020;9:1505.

57. Hadzhieva M, Kirches E, Mawrin C. Review: iron metabolism and the role of iron in neurodegenerative disorders. Neuropathol Appl Neurobiol. 2014;40:240-57.

58. Philpott CC, Patel SJ, Protchenko O. Management versus miscues in the cytosolic labile iron pool: The varied functions of iron chaperones. Biochim Biophys Acta Mol Cell Res. 2020;1867:118830.

59. Feng H, Schorpp K, Jin J, Yozwiak CE, Hoffstrom BG, Decker AM, et al. Transferrin receptor is a specific ferroptosis marker. Cell Rep. 2020;30:3411-3423. e7

60. Shibata Y, Yasui H, Higashikawa K, Kuge Y. Transferrin-based radiolabeled probe predicts the sensitivity of human renal cancer cell lines to ferroptosis inducer erastin. Biochem Biophys Rep. 2021;26:100957.

61. McCullough K, Bolisetty S. Iron homeostasis and ferritin in sepsis-associated kidney injury. Nephron. 2020;144:616-20.

62. Yagoda N, von Rechenberg M, Zaganjor E, Bauer AJ, Yang WS, Fridman DJ, et al. RAS-RAF-MEK-dependent oxidative cell death involving voltage-dependent anion channels. Nature. 2007;447:864-8.

63. Chen L, Li X, Liu L, Yu B, Xue Y, Liu Y. Erastin sensitizes glioblastoma cells to temozolomide by restraining $x C T$ and cystathionine- $\gamma$-lyase function. Oncol Rep. 2015;33:1465-74.

64. Peng W, Zhu Z, Yang Y, Hou J, Lu J, Chen C, et al. N2L, a novel lipoic acid-niacin dimer, attenuates ferroptosis and decreases lipid peroxidation in HT22 cells. Brain Res Bull. 2021;174:250-9.

65. Wu Y, Song J, Wang Y, Wang X, Culmsee C, Zhu C. The potential role of ferroptosis in neonatal brain injury. Front Neurosci. 2019;13:115.

66. Hou K, Shen J, Yan J, Zhai C, Zhang J, Pan JA, et al. Loss of TRIM21 alleviates cardiotoxicity by suppressing ferroptosis induced by the chemotherapeutic agent doxorubicin. EBioMedicine. 2021;69:103456.

67. Gan B. Mitochondrial regulation of ferroptosis. J Cell Biol. 2021;220:e202105043.

68. Niu B, Lei $X, X u Q$, Ju $Y, X u D, M a o ~ L$, et al. Protecting mitochondria via inhibiting VDAC1 oligomerization alleviates ferroptosis in acetaminopheninduced acute liver injury. Cell Biol Toxicol. 2021. https://doi.org/10.1007/ s10565-021-09624-x

69. Wei S, Qiu T, Yao X, Wang N, Jiang L, Jia X, et al. Arsenic induces pancreatic dysfunction and ferroptosis via mitochondrial ROS-autophagy-lysosomal pathway. J Hazard Mater. 2020;384:121390.

70. Sumneang N, Siri-Angkul N, Kumfu S, Chattipakorn SC, Chattipakorn N. The effects of iron overload on mitochondrial function, mitochondrial dynamics, and ferroptosis in cardiomyocytes. Arch Biochem Biophys. 2020;680:108241.

71. Vučković AM, Venerando R, Tibaldi E, Bosello Travain V, Roveri A, Bordin L, et al. Aerobic pyruvate metabolism sensitizes cells to ferroptosis primed by GSH depletion. Free Radic Biol Med. 2021;167:45-53.

72. Yuan H, Li X, Zhang X, Kang R, Tang D. CISD1 inhibits ferroptosis by protection against mitochondrial lipid peroxidation. Biochem Biophys Res Commun. 2016;478:838-44.
73. Hu Z, Zhang H, Yi B, Yang S, Liu J, Hu J, et al. VDR activation attenuate cisplatin induced AKI by inhibiting ferroptosis. Cell Death Dis. 2020;11:73.

74. Liu L, Li L, Li M, Luo Z. Autophagy-dependent ferroptosis as a therapeutic target in cancer. ChemMedChem. 2021;16:2942-50.

75. Hirata Y, Tsunekawa Y, Takahashi M, Oh-Hashi K, Kawaguchi K, Hayazaki M, et al. Identification of novel neuroprotective $\mathrm{N}, \mathrm{N}$-dimethylaniline derivatives that prevent oxytosis/ferroptosis and localize to late endosomes and lysosomes. Free Radic Biol Med. 2021;174:225-35.

76. Debieu S, Solier S, Colombeau L, Versini A, Sindikubwabo F, Forrester A, et al. Small molecule regulators of ferroptosis. Adv Exp Med Biol. 2021;1301:81-121.

77. Kurz T, Eaton JW, Brunk UT. The role of lysosomes in iron metabolism and recycling. Int J Biochem Cell Biol. 2011;43:1686-97.

78. Zhang $\mathrm{X}, \mathrm{Yu} \mathrm{L}, \mathrm{Xu} \mathrm{H}$. Lysosome calcium in ROS regulation of autophagy. Autophagy. 2016;12:1954-5.

79. Buratta S, Tancini B, Sagini K, Delo F, Chiaradia E, Urbanelli L, et al. Lysosomal exocytosis, exosome release and secretory autophagy: the autophagic- and endo-lysosomal systems go extracellular. Int J Mol Sci. 2020;21:2576.

80. Schulze RJ, Krueger EW, Weller SG, Johnson KM, Casey CA, Schott MB, et al. Direct lysosome-based autophagy of lipid droplets in hepatocytes. Proc Natl Acad Sci USA. 2020;117:32443-52.

81. Gao $M$, Monian $P$, Pan $Q$, Zhang W, Xiang J, Jiang X. Ferroptosis is an autophagic cell death process. Cell Res. 2016;26:1021-32.

82. Chen C, Wang D, Yu Y, Zhao T, Min N, Wu Y, et al. Legumain promotes tubular ferroptosis by facilitating chaperone-mediated autophagy of GPX4 in AKI. Cell Death Dis. 2021;12:65.

83. Cybulsky AV. Endoplasmic reticulum stress, the unfolded protein response and autophagy in kidney diseases. Nat Rev Nephrol. 2017;13:681-96.

84. Gagliardi M, Cotella D, Santoro C, Corà D, Barlev NA, Piacentini M, et al. Aldoketo reductases protect metastatic melanoma from ER stress-independent ferroptosis. Cell Death Dis. 2019;10:902.

85. Lee YS, Lee DH, Choudry HA, Bartlett DL, Lee YJ. Ferroptosis-induced endoplasmic reticulum stress: cross-talk between ferroptosis and apoptosis. Mol Cancer Res. 2018;16:1073-6.

86. Yoshida GJ. The interplay between apoptosis and ferroptosis mediated by ER stress. Apoptosis. 2020;25:784-5.

87. Dixon SJ, Patel DN, Welsch M, Skouta R, Lee ED, Hayano M, et al. Pharmacological inhibition of cystine-glutamate exchange induces endoplasmic reticulum stress and ferroptosis. elife 2014;3:e02523.

88. Zhu S, Zhang Q, Sun X, Zeh HJ 3rd, Lotze MT, Kang R, et al. HSPA5 regulates ferroptotic cell death in cancer cells. Cancer Res. 2017;77:2064-77.

89. Xu M, Tao J, Yang Y, Tan S, Liu H, Jiang J, et al. Ferroptosis involves in intestinal epithelial cell death in ulcerative colitis. Cell Death Dis. 2020;11:86.

90. Park EJ, Park YJ, Lee SJ, Lee K, Yoon C. Whole cigarette smoke condensates induce ferroptosis in human bronchial epithelial cells. Toxicol Lett. 2019;303:55-66.

91. Hong SH, Lee DH, Lee YS, Jo MJ, Jeong YA, Kwon WT, et al. Molecular crosstalk between ferroptosis and apoptosis: emerging role of ER stress-induced p53independent PUMA expression. Oncotarget. 2017;8:115164-78.

92. Alborzinia H, Ignashkova TI, Dejure FR, Gendarme M, Theobald J, Wölfl S, et al. Golgi stress mediates redox imbalance and ferroptosis in human cells. Commun Biol. 2018;1:210.

93. Chen X, Kang R, Kroemer G, Tang D. Organelle-specific regulation of ferroptosis. Cell Death Differ. 2021;28:2843-5286.

94. Wu Y, Zhang S, Gong X, Tam S, Xiao D, Liu S, et al. The epigenetic regulators and metabolic changes in ferroptosis-associated cancer progression. Mol Cancer. 2020;19:39.

95. Sasaki K, Yoshida H. Organelle autoregulation-stress responses in the ER, Golgi, mitochondria and lysosome. J Biochem. 2015;157:185-95.

96. Galluzzi L, Bravo-San Pedro JM, Kroemer G. Organelle-specific initiation of cell death. Nat Cell Biol. 2014;16:728-36.

97. Cerri S, Milanese C, Mastroberardino PG. Endocytic iron trafficking and mitochondria in Parkinson's disease. Int J Biochem Cell Biol. 2019;110:70-74.

98. Zhao X, Guo X, Tang X, Zhang H, Wang M, Kong Y, et al. Misregulation of ERGolgi vesicle transport induces ER stress and affects seed vigor and stress response. Front Plant Sci. 2018;9:658.

99. Palmer SN, Chappidi S, Pinkham C, Hancks DC. Evolutionary profile for (host and viral) MLKL indicates its activities as a battlefront for extensive counteradaptation. Mol Biol Evol. 2021;38:5405-22.

100. Zanetti LC, Weinlich R. Necroptosis, the other main caspase-independent cell death. Adv Exp Med Biol. 2021;1301:123-38.

101. Wu F, Shao Q, Cheng Z, Xiong X, Fang K, Zhao Y, et al. Traditional herbal formula Wu-Mei-Wan alleviates TNBS-induced colitis in mice by inhibiting necroptosis through increasing RIPK3 O-GIcNAcylation. Chin Med. 2021;16:78.

102. Zhao Z, Wu J, Xu H, Zhou C, Han B, Zhu H, et al. XJB-5-131 inhibited ferroptosis in tubular epithelial cells after ischemia-reperfusion injury. Cell Death Dis. 2020;11:629. 
103. Müller T, Dewitz C, Schmitz J, Schröder AS, Bräsen JH, Stockwell BR, et al. Necroptosis and ferroptosis are alternative cell death pathways that operate in acute kidney failure. Cell Mol Life Sci. 2017;74:3631-45.

104. Martin-Sanchez D, Ruiz-Andres O, Poveda J, Carrasco S, Cannata-Ortiz P, Sanchez-Niño MD, et al. Ferroptosis, but not necroptosis, is important in nephrotoxic folic acid-induced AKI. JASN. 2017;28:218-29.

105. Belavgeni A, Meyer C, Stumpf J, Hugo C, Linkermann A. Ferroptosis and necroptosis in the kidney. Cell Chem Biol. 2020;27:448-62.

106. Sancho-Martínez SM, López-Novoa JM, López-Hernández FJ. Pathophysiological role of different tubular epithelial cell death modes in acute kidney injury. Clin Kidney J. 2015;8:548-59.

107. Liu J, Kuang F, Kroemer G, Klionsky DJ, Kang R, Tang D. Autophagy-dependent ferroptosis: machinery and regulation. Cell Chem Biol. 2020;27:420-35.

108. Li M, Wang X, Lu S, He C, Wang C, Wang L, et al. Erastin triggers autophagic death of breast cancer cells by increasing intracellular iron levels. Oncol Lett. 2020;20:57.

109. Park E, Chung SW. ROS-mediated autophagy increases intracellular iron levels and ferroptosis by ferritin and transferrin receptor regulation. Cell Death Dis. 2019;10:822.

110. Hou W, Xie Y, Song X, Sun X, Lotze MT, Zeh HJ 3rd, et al. Autophagy promotes ferroptosis by degradation of ferritin. Autophagy. 2016;12:1425-8.

111. Liu J, Guo ZN, Yan XL, Huang S, Ren JX, Luo Y, et al. Crosstalk between autophagy and ferroptosis and its putative role in ischemic stroke. Front Cell Neurosci. 2020;14:577403.

112. Sun $Y$, Chen $P$, Zhai $B$, Zhang $M$, Xiang $Y$, Fang J, et al. The emerging role of ferroptosis in inflammation. Biomed Pharmacother. 2020;127:110108.

113. Tang D, Chen $X$, Kang R, Kroemer G. Ferroptosis: molecular mechanisms and health implications. Cell Res. 2021;31:107-25.

114. Linkermann A, Skouta R, Himmerkus N, Mulay SR, Dewitz C, De Zen F, et al. Synchronized renal tubular cell death involves ferroptosis. Proc Natl Acad Sci USA. 2014;111:16836-41.

115. Martin-Sanchez D, Fontecha-Barriuso M, Martinez-Moreno JM, Ramos AM, Sanchez-Niño MD, Guerrero-Hue $M$, et al. Ferroptosis and kidney disease. Nefrologia. 2020;40:384-94.

116. Bucaloiu ID, Kirchner HL, Norfolk ER, Hartle JE 2nd, Perkins RM. Increased risk of death and de novo chronic kidney disease following reversible acute kidney injury. Kidney Int. 2012;81:477-85.

117. Sato $Y$, Takahashi M, Yanagita M. Pathophysiology of AKI to CKD progression. Semin Nephrol. 2020;40:206-15.

118. Sui M, Xu D, Zhao W, Lu H, Chen R, Duan Y, et al. CIRBP promotes ferroptosis by interacting with ELAVL1 and activating ferritinophagy during renal ischaemiareperfusion injury. J Cell Mol Med. 2021;25:6203-16.

119. Sha W, Hu F, Xi Y, Chu Y, Bu S. Mechanism of ferroptosis and its role in type 2 diabetes mellitus. J Diabetes Res. 2021;2021:9999612.

120. Wang WJ, Jiang $X$, Gao CC, Chen ZW. Salusin- $\beta$ participates in high glucose-induced HK-2 cell ferroptosis in a Nrf-2-dependent manner. Mol Med Rep. 2021;24:674.

121. Wang J, Liu Y, Wang Y, Sun L. The cross-link between ferroptosis and kidney diseases. Oxid Med Cell Longev. 2021;2021:6654887.

122. Fu Y, Tang C, Cai J, Chen G, Zhang D, Dong Z. Rodent models of AKI-CKD transition. Am J Physiol Ren Physiol. 2018;315:F1098-f1106.

123. Newton K, Dugger DL, Maltzman A, Greve JM, Hedehus M, Martin-McNulty B, et al. RIPK3 deficiency or catalytically inactive RIPK1 provides greater benefit than MLKL deficiency in mouse models of inflammation and tissue injury. Cell Death Differ. 2016;23:1565-76.

124. Linkermann A, Bräsen JH, Darding M, Jin MK, Sanz AB, Heller JO, et al. Two independent pathways of regulated necrosis mediate ischemia-reperfusion injury. Proc Natl Acad Sci USA. 2013;110:12024-9.

125. Choi N, Whitlock R, Klassen J, Zappitelli M, Arora RC, Rigatto C, et al. Early intraoperative iron-binding proteins are associated with acute kidney injury after cardiac surgery. J Thorac Cardiovasc Surg. 2019;157:287-297. e2

126. Su L, Jiang $X$, Yang C, Zhang J, Chen B, Li Y, et al. Pannexin 1 mediates ferroptosis that contributes to renal ischemia/reperfusion injury. J Biol Chem. 2019:294:19395-404.

127. Smeland E, Fuskevåg OM, Nymann K, Svendesn JS, Olsen R, Lindal S, et al. Highdose 7-hydromethotrexate: acute toxicity and lethality in a rat model. Cancer Chemother Pharm. 1996;37:415-22.

128. Ozkok A, Edelstein CL. Pathophysiology of cisplatin-induced acute kidney injury. Biomed Res Int. 2014;2014:967826.

129. Xu Y, Ma H, Shao J, Wu J, Zhou L, Zhang Z, et al. A role for tubular necroptosis in Cisplatin-induced AKI. JASN. 2015;26:2647-58.

130. Baliga R, Zhang Z, Baliga M, Ueda N, Shah SV. In vitro and in vivo evidence suggesting a role for iron in cisplatin-induced nephrotoxicity. Kidney Int. 1998;53:394-401.
131. Lu Y, Cederbaum Al. Cisplatin-induced hepatotoxicity is enhanced by elevated expression of cytochrome P450 2E1. Toxicol Sci. 2006;89:515-23.

132. Ikeda $Y$, Hamano $H$, Horinouchi $Y$, Miyamoto L, Hirayama $T$, Nagasawa $H$, et al. Role of ferroptosis in cisplatin-induced acute nephrotoxicity in mice. J Trace Elem Med Biol. 2021;67:126798.

133. Scurt FG, Bose K, Canbay A, Mertens PR, Chatzikyrkou C. [Acute kidney injury following acute pancreatitis (AP-AKI):definition, pathophysiology, diagnosis and therapy]. Z Gastroenterol. 2020;58:1241-66.

134. Ma D, Li C, Jiang P, Jiang Y, Wang J, Zhang D. Inhibition of ferroptosis attenuates acute kidney injury in rats with severe acute pancreatitis. Dig Dis Sci. 2021;66:483-92.

135. Chen Y, Fan H, Wang S, Tang G, Zhai C, Shen L. Ferroptosis: a novel therapeutic target for ioschemia-reperfusion injury. Front Cell Dev Biol. 2021;9:688605.

136. Song Q, Peng S, Sun Z, Heng X, Zhu X. Temozolomide drives ferroptosis via a DMT1-dependent pathway in glioblastoma cells. Yonsei Med J. 2021;62:843-9.

137. Chen B, Wang H, Lv C, Mao C, Cui Y. Long non-coding RNA H19 protects against intracerebral hemorrhage injuries via regulating microRNA-106b-5p/acyl-CoA synthetase long chain family member 4 axis. Bioengineered. 2021;12:4004-15.

138. Dong LH, Huang JJ, Zu P, Liu J, Gao X, Du JW, et al. CircKDM4C upregulates P53 by sponging hsa-let-7b-5p to induce ferroptosis in acute myeloid leukemia. Environ Toxicol. 2021;36:1288-302.

139. Wen L, Zhou T, Jiang $Y$, Gong L, Yang B. Identification of prenylated phenolics in mulberry leaf and their neuroprotective activity. Phytomedicine. 2021;90:153641.

140. Han F, Li S, Yang Y, Bai Z. Interleukin-6 promotes ferroptosis in bronchial epithelial cells by inducing reactive oxygen species-dependent lipid peroxidation and disrupting iron homeostasis. Bioengineered. 2021;12:5279-88.

141. Homma T, Kobayashi S, Conrad M, Konno H, Yokoyama C, Fujii J. Nitric oxide protects against ferroptosis by aborting the lipid peroxidation chain reaction. Nitric Oxide. 2021;115:34-43.

142. Martinez AM, Kim A, Yang WS. Detection of ferroptosis by BODIPYTM $581 / 591$ C11. Methods Mol Biol. 2020;2108:125-30.

143. Cheng G, Zielonka M, Dranka B, Kumar SN, Myers CR, Bennett B, et al. Detection of mitochondria-generated reactive oxygen species in cells using multiple probes and methods: potentials, pitfalls, and the future. J Biol Chem. 2018;293:10363-80.

144. Yuan Y, Zhai Y, Chen J, Xu X, Wang H. Kaempferol ameliorates oxygen-glucose deprivation/reoxygenation-induced neuronal ferroptosis by activating Nrf2/ SLC7A11/GPX4 axis. Biomolecules. 2021;11:923.

145. Li MY, Dai XH, Yu XP, Zou W, Teng W, Liu P, et al. Scalp acupuncture protects against neuronal ferroptosis by activating the p62-Keap1-Nrf2 pathway in rat models of intracranial haemorrhage. J Mol Neurosci. 2022;72:82-96.

146. Lipper $\mathrm{CH}$, Stofleth JT, Bai F, Sohn YS, Roy S, Mittler R, et al. Redox-dependent gating of VDAC by mitoNEET. Proc Natl Acad Sci USA. 2019;116:19924-9.

147. Chen D, Chu B, Yang X, Liu Z, Jin Y, Kon N, et al. iPLA2 $\beta$-mediated lipid detoxification controls p53-driven ferroptosis independent of GPX4. Nat Commun. 2021;12:3644.

148. Lei G, Zhang Y, Hong T, Zhang X, Liu X, Mao C, et al. Ferroptosis as a mechanism to mediate p53 function in tumor radiosensitivity. Oncogene. 2021;40:3533-47.

149. Chen L, Xie J. Ferroptosis-suppressor-protein 1: a potential neuroprotective target for combating ferroptosis. Mov Disord. 2020;35:400.

150. He L, Liu YY, Wang K, Li C, Zhang W, Li ZZ, et al. Tanshinone IIA protects human coronary artery endothelial cells from ferroptosis by activating the NRF2 pathway. Biochem Biophys Res Commun. 2021;575:1-7.

151. Bersuker K, Hendricks JM, Li Z, Magtanong L, Ford B, Tang PH, et al. The CoQ oxidoreductase FSP1 acts parallel to GPX4 to inhibit ferroptosis. Nature. 2019:575:688-92.

152. Sun X, Ou Z, Xie M, Kang R, Fan Y, Niu X, et al. HSPB1 as a novel regulator of ferroptotic cancer cell death. Oncogene. 2015;34:5617-25.

153. Wen Y, Chen H, Zhang L, Wu M, Zhang F, Yang D, et al. Glycyrrhetinic acid induces oxidative/nitrative stress and drives ferroptosis through activating NADPH oxidases and iNOS, and depriving glutathione in triple-negative breast cancer cells. Free Radic Biol Med. 2021;173:41-51.

154. Zhang L, Wang F, Li D, Yan Y, Wang H. Transferrin receptor-mediated reactive oxygen species promotes ferroptosis of KGN cells via regulating NADPH oxidase 1/PTEN induced kinase 1/acyl-CoA synthetase long chain family member 4 signaling. Bioengineered. 2021;12:4983-94.

155. Yao $F$, Cui $X$, Zhang $Y$, Bei $Z$, Wang $H$, Zhao $D$, et al. Iron regulatory protein 1 promotes ferroptosis by sustaining cellular iron homeostasis in melanoma. Oncol Lett. 2021;22:657.

156. Hayano M, Yang WS, Corn CK, Pagano NC, Stockwell BR. Loss of cysteinyl-tRNA synthetase (CARS) induces the transsulfuration pathway and inhibits ferroptosis induced by cystine deprivation. Cell Death Differ. 2016:23:270-8.

157. Ye Q, Zeng C, Luo C, Wu Y. Ferrostatin-1 mitigates cognitive impairment of epileptic rats by inhibiting P38 MAPK activation. Epilepsy Behav. 2020;103:106670. 
158. Nakamura T, Naguro I, Ichijo H. Iron homeostasis and iron-regulated ROS in cell death, senescence and human diseases. Biochim Biophys Acta Gen Subj. 2019;1863:1398-409.

159. Dangol S, Nguyen NK, Singh R, Chen Y, Wang J, Lee HG, et al. Mitogen-activated protein kinase OsMEK2 and OsMPK1 signaling is required for ferroptotic cell death in rice-Magnaporthe oryzae interactions. Front Plant Sci. 2021;12:710794.

160. Fang $Y$, Chen X, Tan Q, Zhou H, Xu J, Gu Q. Inhibiting ferroptosis through disrupting the NCOA4-FTH1 interaction: a new mechanism of action. ACS Cent Sci. 2021;7:980-9.

161. Yang A, Wang L, Jiang K, Lei L, Li H. Nuclear receptor coactivator 4-mediated ferritinophagy drives proliferation of dental pulp stem cells in hypoxia. Biochem Biophys Res Commun. 2021;554:123-30.

162. Santana-Codina N, Gikandi A, Mancias JD. The role of NCOA4-mediated ferritinophagy in ferroptosis. Adv Exp Med Biol. 2021;1301:41-57.

163. Stoyanovsky DA, Tyurina YY, Shrivastava I, Bahar I, Tyurin VA, Protchenko O, et al. Iron catalysis of lipid peroxidation in ferroptosis: regulated enzymatic or random free radical reaction? Free Radic Biol Med. 2019;133:153-61.

164. Clemente SM, Martínez-Costa OH, Monsalve M, Samhan-Arias AK. Targeting lipid peroxidation for Cancer Treatment. Molecules. 2020;25:5144.

165. Yang J, Zhou Y, Xie S, Wang J, Li Z, Chen L, et al. Metformin induces ferroptosis by inhibiting UFMylation of SLC7A11 in breast cancer. J Exp Clin Cancer Res. 2021;40:206

166. Feng $L$, Zhao K, Sun L, Yin X, Zhang J, Liu C, et al. SLC7A11 regulated by NRF2 modulates esophageal squamous cell carcinoma radiosensitivity by inhibiting ferroptosis. J Transl Med. 2021;19:367.

167. Zhang X, Zheng C, Gao Z, Chen H, Li K, Wang L, et al. SLC7A11/xCT prevents hypertrophy by inhibiting ferroptosis. Cardiovasc Drugs Ther. 2021. https://doi. org/10.1007/s10557-021-07220-z.

168. Wenzel SE, Tyurina YY, Zhao J, St Croix CM, Dar HH, Mao G, et al. PEBP1 wardens ferroptosis by enabling lipoxygenase generation of lipid death signals. Cell 2017;171:628-641.e26.

169. Ding C, Ding $X$, Zheng J, Wang B, Li Y, Xiang H, et al. miR-182-5p and miR-378a$3 p$ regulate ferroptosis in I/R-induced renal injury. Cell Death Dis. 2020;11:929.

170. Deng F, Sharma I, Dai Y, Yang M, Kanwar YS. Myo-inositol oxygenase expression profile modulates pathogenic ferroptosis in the renal proximal tubule. J Clin Invest. 2019;129:5033-49.

171. Avunduk MC, Yurdakul T, Erdemli E, Yavuz A. Prevention of renal damage by alpha tocopherol in ischemia and reperfusion models of rats. Urol Res. 2003;31:280-5.

172. Zager RA. Combined mannitol and deferoxamine therapy for myohemoglobinuric renal injury and oxidant tubular stress. Mechanistic and therapeutic implications. J Clin Invest. 1992;90:711-9.

173. Doll S, Proneth B, Tyurina YY, Panzilius E, Kobayashi S, Ingold I, et al. ACSL4 dictates ferroptosis sensitivity by shaping cellular lipid composition. Nat Chem Biol. 2017:13:91-98.

174. Lu Q, Wang M, Gui Y, Hou Q, Gu M, Liang Y, et al. Rheb1 protects against cisplatin-induced tubular cell death and acute kidney injury via maintaining mitochondrial homeostasis. Cell Death Dis. 2020;11:364.

175. Jiang GP, Liao YJ, Huang LL, Zeng XJ, Liao XH. Effects and molecular mechanism of pachymic acid on ferroptosis in renal ischemia reperfusion injury. Mol Med Rep.2021;23:63.

176. Tang Y, Luo H, Xiao Q, Li L, Zhong X, Zhang J, et al. Isoliquiritigenin attenuates septic acute kidney injury by regulating ferritinophagy-mediated ferroptosis. Ren Fail. 2021;43:1551-60.

177. Wang Y, Quan F, Cao Q, Lin Y, Yue C, Bi R, et al. Quercetin alleviates acute kidney injury by inhibiting ferroptosis. J Adv Res. 2021;28:231-43.

\section{ACKNOWLEDGEMENTS}

This work was supported by grants from the National Natural Science Foundation of China (82100763), Hubei Provincial Natural Science Foundation of China (2021CFB090), the Fundamental Research Funds for the Central University (2042021kf0150 and 2042020kf0137), Zhongnan Hospital of Wuhan University Science, Technology and Innovation Seed Fund, Project (cxpy2020027 and znpy2019036). Excellent Doctor (Post), Zhongnan Hospital of Wuhan University (ZNYB2020009), Zhongnan Hospital of Wuhan University Science, Technology and Innovation Seed Fund, Project znpy2019036 and znpy2017044. Hubei Province Health and Family Planning Scientific Research Project (WJ2019MB103). The Clinical Research Project for Wu Jieping Medical Foundation (320.6750.19089-58). The Research Fund from Medical Sci-Tech Innovation Platform of Zhongnan Hospital, Wuhan University (PTXM2020028).

\section{AUTHOR CONTRIBUTIONS}

Cheng Yuan conceived and designed the study. Lihua Ni wrote the manuscript. All authors revised and approved the submitted version.

\section{COMPETING INTERESTS}

The authors declare no competing interests.

\section{ADDITIONAL INFORMATION}

Supplementary information The online version contains supplementary material available at https://doi.org/10.1038/s41419-022-04628-9.

Correspondence and requests for materials should be addressed to Cheng Yuan or Xiaoyan Wu.

Reprints and permission information is available at http://www.nature.com/ reprints

Publisher's note Springer Nature remains neutral with regard to jurisdictional claims in published maps and institutional affiliations.

\footnotetext{
cc) (i)

Open Access This article is licensed under a Creative Commons Attribution 4.0 International License, which permits use, sharing, adaptation, distribution and reproduction in any medium or format, as long as you give appropriate credit to the original author(s) and the source, provide a link to the Creative Commons license, and indicate if changes were made. The images or other third party material in this article are included in the article's Creative Commons license, unless indicated otherwise in a credit line to the material. If material is not included in the article's Creative Commons license and your intended use is not permitted by statutory regulation or exceeds the permitted use, you will need to obtain permission directly from the copyright holder. To view a copy of this license, visit http://creativecommons. org/licenses/by/4.0/.
}

(c) The Author(s) 2022 